\title{
Retrospective analysis of prognostic factors in patients of papillary thyroid microcarcinoma
}

\author{
Tinghai Xiang ${ }^{1}$, Wenyan Yan ${ }^{2}$ and Longan Zhou ${ }^{1}$ \\ ${ }^{1}$ Department of General Surgery, Binzhou People's Hospital, Binzhou 256610, Shandong, China \\ ${ }^{2}$ Section II, Department of Neurology, Binzhou People's Hospital, Binzhou 256610, Shandong, China \\ Correspondence to: Tinghai Xiang, email: xiangtinghai@126.com \\ Keywords: papillary thyroid microcarcinoma; central lymph node dissection; therapy; prognosis \\ Received: January 06, $2018 \quad$ Accepted: May 13, $2018 \quad$ Published: October 30, 2018 \\ Copyright: Xiang et al. This is an open-access article distributed under the terms of the Creative Commons Attribution License 3.0 \\ (CC BY 3.0), which permits unrestricted use, distribution, and reproduction in any medium, provided the original author and source \\ are credited.
}

\section{ABSTRACT}

We performed a retrospective chart review of $\mathbf{2 4 5}$ patients with papillary thyroid microcarcinoma (PTMC) to define factors linked to central lymph node metastasis and thus prognosis. Univariate and multivariate analyses showed that being male $(p<0.001)$, age $<45$ years at diagnosis $(p=0.045)$, maximum tumor size $>5 \mathrm{~mm}$ $(p=0.030)$, multifocal tumor $(p=0.040)$ and tumor envelope invasion $(p<0.001)$ were all independent risk factors for central compartment lymph node metastasis. Unifocal lesions at the thyroid gland's upper pole, middle and lower pole, had lymph node metastasis rates of $22.7 \%, 14.0 \%$ and $35.0 \%$, respectively $(p=0.048)$. The rate of central lymph node metastasis was much higher when there was bilateral thyroid involvement than with multifocal unilateral lesions $(58.6 \%$ vs $37.5 \% ; p=0.040)$. These results suggest that for patients at low risk of central lymph node metastasis, unilateral thyroid lobe and isthmus resection is sufficient. However, for patients at high risk of central lymph node metastasis, central lymph node dissection increases the likelihood of complete tumor excision.

\section{INTRODUCTION}

Papillary thyroid microcarcinomas (PTMCs) are small papillary thyroid carcinomas with maximum diameters less than $1.0 \mathrm{~cm}$. Cervical lymph node metastasis is the most frequent cause of long-term recurrence, with a rate of about $12 \%$ [1]. Although the overall prognosis of PRMC patients is good, some PTMCs are prone to glandular invasion and lymph node metastasis. Consequently, if the treatment is not complete, the risk of recurrence can be as high as $20-30 \%$ and ultimately affect the patient prognosis $[2,3]$. In the present study, we retrospectively reviewed the surgical treatment of PTMC and analyzed factors that could affect prognosis, including metastasis of central compartment lymph nodes.

\section{RESULTS}

Univariate analysis of cervical lymph node metastasis

Univariate analyses showed that the risk factors for cervical lymph node metastasis in PTMC patients included being male, the diagnosed at $<45$ years of age, maximum tumor maximum size $>5 \mathrm{~mm}$, multifocal tumor, and tumor envelope invasion (Table 1). Hashimoto's thyroiditis was not a risk factor for cervical lymph node metastasis.

Multivariate analysis of cervical lymph node metastasis

We next performed a multivariate analysis using the significant indicators from the univariate analyses 
Table 1: Univariate analyses of central lymph node metastasis

\begin{tabular}{|c|c|c|c|c|c|}
\hline Parameters & $\begin{array}{c}\text { CLNM(-) } \\
(n=159)\end{array}$ & $\begin{array}{c}\operatorname{CLNM}(+) \\
(n=86)\end{array}$ & $\begin{array}{c}\text { Metastasis rate } \\
(\%)\end{array}$ & $\chi^{2}$ & $\bar{p}$ \\
\hline Gender & & & & 27.570 & 0.000 \\
\hline Male & 45 & 54 & 54.5 & & \\
\hline Female & 114 & 32 & 21.9 & & \\
\hline Age (years) & & & & 4.447 & 0.035 \\
\hline$<45$ & 68 & 25 & 26.9 & & \\
\hline$\geq 45$ & 91 & 61 & 40.1 & & \\
\hline Tumor size (mm) & & & & 4.853 & 0.028 \\
\hline$>5$ & 88 & 60 & 40.5 & & \\
\hline$\leq 5$ & 71 & 26 & 26.8 & & \\
\hline Capsular invasion & & & & 20.133 & 0.000 \\
\hline Yes & 38 & 45 & 54.2 & & \\
\hline No & 121 & 41 & 25.3 & & \\
\hline PTMC with HT & & & & 0.399 & 0.528 \\
\hline Yes & 37 & 17 & 31.5 & & \\
\hline No & 122 & 62 & 33.7 & & \\
\hline \multicolumn{6}{|l|}{ Tumor location } \\
\hline Single focus & & & & 6.090 & 0.048 \\
\hline Upper pole & 34 & 10 & 22.7 & & \\
\hline Middle third & 37 & 6 & 14.0 & & \\
\hline Lower pole & 39 & 21 & 35.0 & & \\
\hline Multifocal & & & & 4.224 & 0.040 \\
\hline Unilateral & 25 & 15 & 37.5 & & \\
\hline Bilateral & 24 & 34 & 58.6 & & \\
\hline
\end{tabular}

with a two-classification logistic regression model. This analysis showed that diagnosis of PTMC at $<45$ years of age $(p=0.045)$, being male $(p<0.001)$, maximum tumor diameter $>5 \mathrm{~mm}(p=0.030)$, and tumor envelope invasion $(P<0.001)$ were all independent risk factors for cervical lymph node metastasis. Our findings also indicated that the lymph node metastasis rates for single lesions located at the upper pole, middle and lower pole of the thyroid gland $(22.7 \%, 14.0 \%$ and $35.0 \%$, respectively) differ significantly $(p<0.05)$. When the tumor was located in the bilateral thyroid glands, the rate of central lymph node metastasis was much higher than with multifocal unilateral lesions $(58.6 \%$ vs $37.5 \% ; p=0.040)$ (Table 2$)$.

\section{Relationship between numbers of lesions and central lymph node metastasis}

Sixty percent of patients presented with a single tumor, while $40 \%$ had 2 or more tumors. Single factor analysis showed that the number of tumors was related to lymph node metastasis $(p=0.040)$, and that the lymph node metastasis was nearly twice as likely to occur in patients with multifocal tumors $(50.0 \%$ vs $25.2 \% ; p=0.021)$ (Table 1$)$.

\section{DISCUSSION}

\section{The prognosis of patients with PTMC}

The detection rate of PTMC has been steadily increasing thanks to improvements in diagnostic ultrasound technology and the wide application of ultrasound-guided fine needle aspiration biopsy $[4,5]$. Although it was generally believed that the majority of PTMCs progress slowly and that patient prognosis is good, some patients exhibit central, and even lateral, cervical lymph node metastasis at the time of surgical treatment. The reported central lymph node metastasis rate in PTMC ranges from $24.1 \%$ to $64.1 \%$ [6-8]. In our study, central lymph node metastasis was detected in $35.1 \%$ of cases. Single and multiple factor regression analyses indicated that being male, age $>45$ years, tumor capsule/gland external invasion, and diameter $>5 \mathrm{~mm}$ are all risk factors for lymph node metastasis in these PTMC patients, which is consistent with earlier reports [9-11].

At present, the relationship between lesion location and lymph node metastasis remains controversial. The presence of a unifocal PTMC at the lower pole of 
Table 2: Multivariate logistic regression analysis of central lymph node metastasis

\begin{tabular}{lcccccc}
\hline Parameters & B & S.E. & Wald & df & $\boldsymbol{p}$ & 95\% CL \\
\hline Gender & 0.634 & 0.142 & 27.014 & 1 & 0.000 & $1.230 \sim 2.385$ \\
Age & -0.501 & 0.099 & 3.185 & 1 & 0.045 & $0.426 \sim 1.018$ \\
Tumor size & 0.836 & 0.126 & 4.015 & 1 & 0.030 & $0.363 \sim 0.658$ \\
Capsular invasion & -0.510 & 0.143 & 18.135 & 1 & 0.000 & $0.524 \sim 0.897$ \\
Multifocal & & & 1.988 & 1 & 0.040 & \\
$\quad 1$ & 0.162 & 0.186 & 0.902 & 1 & 0.080 & $0.598 \sim 0.721$ \\
$\quad 2$ & 0.575 & 0.301 & 0.719 & 1 & 0.010 & $0.951 \sim 1.827$ \\
Bilateral & 0.461 & 0.197 & 4.250 & 1 & 0.021 & $1.321 \sim 2.459$ \\
Constant & -0.523 & 0.419 & 0.234 & 1 & 0.459 & - \\
\hline
\end{tabular}

the thyroid gland increases the likelihood of central compartment lymph node metastasis. In cases of multifocal PTMC, the rate of metastasis to central region lymph nodes is lower when only one side of the thyroid is involved. However, the current consensus is that multifocal PTMCs present a high risk for lymph node metastasis $[12,13]$.

\section{Treatment of PTMC}

There was still no consensus as to whether preventive CLND should be a routine part of PTMC treatment. Whereas routine CLND in "not recommended" in the 2015 edition of the American Thyroid Association management guidelines [14], 2016 guidelines for the diagnosis and treatment of PTMC from the Chinese Cancer Society advise "routine prophylactic CLND under the premise of technical support" [12].

As metastatic central compartment lymph nodes are usually small, the clavicle and manubrium sterni often prevent satisfactory detection of metastatic nodes using ultrasound $[15,16]$. In the present study, the sensitivities of ultrasound and intraoperative diagnosis was low, and even when the two methods are combined the sensitivity was only $32 \%$, which is not sufficient to guide the surgeons as to extent of operation. Central lymph node metastasis can readily progress to invasion of the recurrent laryngeal nerve, trachea or esophagus, and even the carotid common artery, which reduces curative potential of operation. The most common occurrence of local residual tumor is in the central region [17]. This makes it a tricky task for even the most experienced surgeon to protect the parathyroid and recurrent laryngeal nerve during the second surgery.

For decades the standard treatment for PTMC has been total thyroidectomy with postoperative radioactive iodine (RAI) treatment. For PTMC invading structures outside the thyroid gland, cervical lymph node metastasis or multiple lesions, postoperative RAI treatment did not reduce the recurrence rate $[18,19]$. RAI may cause temporary or permanent damage to the salivary and lacrimal glands and increase the risk of secondary carcinoma in patients [20]. For low-risk patients with metastasis of central compartment lymph nodes, unilateral thyroidectomy thought to be was the best approach [21]. In a large-scale study of 23,605 patients with differentiated thyroid cancer, the total survival rate did not significantly differ between total and unilateral thyroidectomy, but CLND can significantly reduce disease-free survival [22]. In one large study, lymph node metastasis was predictive of adverse outcomes, but the 14-year survival rates were $82 \%$ and $79 \%$, respectively, among patients without and with lymph node metastasis [23]. This suggests CLND improves the survival rate among PTMC patients with central lymph node metastasis. In the present study, there were no instances of distant metastasis or death among patients at low risk of central lymph node metastasis treated with unilateral thyroid lobe and isthmus resection, suggesting that approach is sufficient in such cases. For patients at high-risk of central lymph node metastasis, we suggest resection of the thyroid lobe and simultaneous ipsilateral CLND.

The main reason not to recommend simultaneous CLND is the potential increase in postoperative complications, especially injury to the parathyroid gland and recurrent laryngeal nerve during secondary operations. With total thyroidectomy and bilateral CLND, there is an increased risk of parathyroid injury and laryngeal recurrent nerve palsy. On the other hand, with careful operative procedures, including the use of new techniques such as nanocarbon staining, there need not be a significant increase in the incidence of postoperative complications [24].

\section{PATIENTS AND METHODS}

\section{Patient demographics}

We collected and retrospectively analyzed the clinical data from 245 patients with PTMC treated between January 2011 and December 2016 in the Department of General Surgery, Binzhou People's Hospital. The criteria for inclusion in the study were: (1) initial surgery for 
Table 3: Clinicopathological characteristics of patients with papillary thyroid microcarcinoma

\begin{tabular}{|c|c|c|}
\hline Parameters & Cases & $\%$ \\
\hline \multicolumn{3}{|l|}{ Gender } \\
\hline Male & 99 & 40.4 \\
\hline Female & 146 & 59.6 \\
\hline \multicolumn{3}{|l|}{ Age (years) } \\
\hline$<45$ & 93 & 38.0 \\
\hline$\geq 45$ & 152 & 62.0 \\
\hline \multicolumn{3}{|c|}{ Tumor size $(\mathrm{mm})$} \\
\hline$>5$ & 148 & 60.4 \\
\hline$\leq 5$ & 97 & 39.6 \\
\hline \multicolumn{3}{|c|}{ Capsular invasion } \\
\hline Yes & 83 & 33.9 \\
\hline No & 162 & 66.1 \\
\hline \multicolumn{3}{|c|}{ PTMC with HT } \\
\hline Yes & 54 & 22.0 \\
\hline No & 184 & 78.0 \\
\hline \multicolumn{3}{|l|}{ Multifocal } \\
\hline Yes & 98 & 40.0 \\
\hline No & 147 & 60.0 \\
\hline \multicolumn{3}{|l|}{ Bilateralism } \\
\hline Yes & 58 & 23.6 \\
\hline No & 187 & 76.4 \\
\hline \multicolumn{3}{|l|}{ CLNM } \\
\hline Yes & 59 & 24.1 \\
\hline No & 186 & 75.9 \\
\hline
\end{tabular}

Abbreviations: PTMC: papillary thyroid microcarcinoma; HT: Hashimoto's thyroiditis; CLNM: central lymph node metastasis.

thyroid cancer; (2) postoperative pathology confirming the lesion to be PTMC; (3) unilateral or total thyroidectomy with simultaneous central lymph node excision; and (4) availability of complete clinical and pathological data. Exclusion criteria were: (1) PTMC with distant metastasis: (2) other types of thyroid cancer, such as medullary cancer; and (3) carcinoma of more $1.0 \mathrm{~cm}$ diameter within multifocal PTMC.

A total of 245 cases were included in this study, including 99 males and 146 females. The average age was $48.5 \pm 9.8$ years (range: $20-81$ years), with 93 patients $<45$ years old (38.0\%) and 152 patients $\geq 45$ years old $(62.0 \%)$. The average tumor size was $0.57 \pm 0.27 \mathrm{~cm}$. There were 147 cases $(60.0 \%)$ of single focus PTMC and 98 cases $(40.0 \%)$ of multifocal PTMC, among which there were 58 cases $(23.3 \%)$ with bilateral tumors. There were 83 cases $(33.9 \%)$ with invasion of the glandular membrane and 86 cases (35.1\%) with central lymph node metastasis (Table 3).

\section{Treatment protocols}

All PTMC cases were treated surgically. When nodules were in the unilateral thyroid, only the affected thyroid lobe, its isthmus, and the ipsilateral central lymph nodes were resected. When nodules were detected in both sides of the thyroid gland, total thyroidectomy with bilateral resection of central lymph nodes was performed.

According to the 2002 edition of the American Academy of Otolaryngology-Head and Neck Surgery [25], the extent of central lymph node dissection (CLND) should be as follows: the upper boundary is the lower margin of the hyoid bone; the lower boundary reaches the sternum; the outside edge is the medial carotid sheath, including the near or distal tracheal lymph node and the anterior laryngeal lymph node. Transient hypocalcemia (blood calcium $<2.0 \mathrm{mmol} / \mathrm{L}$ ) was defined as present only within $24 \mathrm{~h}$ after surgery. Thirty minutes after injecting nanocarbon into the thyroid, the central region lymph nodes and thyroid gland stained black were carefully dissected so as to protect the parathyroid. Thyrotropic hormone inhibitor was administered postoperatively to all patients.

\section{Statistical analysis}

Continuous and normally distributed variables are presented as the mean \pm standard deviation. Univariate 
analyses were done using the chi-square test, while logistic regression was used for multivariate analysis. All analyses were performed using PASW statistics 18.0 software. Values of $p<0.05$ were considered significant.

\section{CONFLICTS OF INTEREST}

The authors declare that they have no conflicts of interest.

\section{REFERENCES}

1. Kim JY, Jung EJ, Park T, Jeong SH, Jeong CY, Ju YT, Lee YJ, Hong SC, Choi SK, Ha WS. Impact of tumor size on subclinical central lymph node metastasis in papillary thyroidmicrocarcinoma depends on age. World J Surg Oncol. 2015; 13:88.

2. Kim E, Choi JY, Koo do H, Lee KE, Youn YK. Differences in the characteristics of papillary thyroid mierocareinoma $\leq 5 \mathrm{~mm}$ and $>5 \mathrm{~mm}$ in diameter. Head Neck. 2015; 37:694-697.

3. Cappelli C, Castellano M, Braga M, Gandossi E, Pirola I, De Martino E, Agosti B, Rosei EA. Aggressiveness and outcome of papillary thyroid carcinoma (PTC) versus microcarcinoma (PMC):amono-institutional experience. J Surg Oncol. 2007; 95:555-560.

4. Mazzaferri EL, Sipos J. Should all patients with subcentimeter thyroid nodules undergo fine-needle aspiration biopsy and preoperative neck ultrasonography to define the extent of tumor invasion? Thyroid. 2008; 18:597-602.

5. Yu Y, Gao M. Research progress in the surgical treatment of papillary thyroid microcarcinoma. Chin J Clin Oncol. 2015; 42:487-490.

6. Zhang L, Wei WJ, Ji QH, Zhu YX, Wang ZY, Wang Y, Huang CP, Shen Q, Li DS, Wu Y. Risk factors for neck nodal metastasis in papillary thyroid microcarcinoma: a study of 1066 patients. J Clin Endocrinol Metab. 2012; 97:1250-1257.

7. Guo Y, Liu Z, Yu P, Liu C, Ming J, Zhang N, Yusufu M, Chen $C$, Huang $T$. Using foci number to predict central lymph node metastases of papillary thyroid microcarcinomas with multifocality. Int J Clin Exp Med. 2015; 8:9925-9930.

8. Siddiqui S, White MG, Antic T, Grogan RH, Angelos P, Kaplan EL, Cipriani NA. Clinical and pathologic predictors of lymph node metastasis and recurrence in papillary thyroid micmcarcinoma. Thyroid. 2016; 26:807-815.

9. Lee YS, Shin SC, Lim YS, Lee JC, Wang SG, Son SM, Kim IJ, Lee BJ. Tumor location-dependent skip lateral cervical lymph node metastasis in papillary thyroid cancer. Head Neck. 2014; 36:887-891.

10. Yan H, Zhou X, Jin H, Li X, Zheng M, Ming X, Wang R, Liu J. A study on central lymph node metastasis in 543
cN0 papillary thyroid carcinoma patients. Int J Endocrinol. 2016; 2016:1878194.

11. Vasileiadis I, Karakostas E, Charitoudis G, Stavrianaki A, Kapetanakis S, Kouraklis G, Karatzas T. Papillary thyroid microcarcinoma: clinicopathological characteristics and implications for treatment in 276 patients. Eur J Clin Invest. 2012; 42:657-664.

12. Committee on thyroid cancer of Chinese cancer society. Diagnosis and treatment of papillary thyroid microcarcinoma in China (2016 edition). Chinese Tumor Clinic. 2016; 43:405-411.

13. Xu D, Lv X, Wang S, Dai W. Risk factors for predicting central lymph node metastasis in papillary thyroid microcarcinoma. Int J Clin Exp Pathol. 2014; 7:6199-6205.

14. Haugen BR, Alexander EK, Bible KC, Doherty GM, Mandel SJ, Nikiforov YE, Pacini F, Randolph GW, Sawka AM, Schlumberger M, Schuff KG, Sherman SI, Sosa JA, et al. 2015 American Thyroid Association Management Guidelines for Adult Patients with Thyroid Nodules and Differentiated Thyroid Cancer: The American Thyroid Association Guidelines Task Force on Thyroid Nodules and Differentiated Thyroid Cancer. Thyroid. 2016; 26:1-133.

15. Robbins KT, Shaha AR, Medina JE, Califano JA, Wolf GT, Ferlito A, Som PM, Day TA; Committee for Neck Dissection Classification, American Head and Neck Society. Consensus statement on the classification and term inology of neck dissection. Arch Otolaryngol Head Neck Surg. 2008;134:536-538.

16. Shim MJ, Roh JL, Gong G, Choi KJ, Lee JH, Cho SH, Nam SY, Kim SY. Preoperative detection and predictors of level V lymph node metastasis in patients with papillary thyroid carcinoma. Br J Surg. 2013; 100:497-503.

17. Sywak M, Cornford L, Roach P, Stalberg P, Sidhu S, Delbridge L. Routine ipsilateral level VI lymphadenectomy reduces postoperative thyroglobulin levels in papillary thyroid cancer. Surgery. 2006; 140:1005-1007.

18. Ross DS, Litofsky D, Ain KB, Bigos T, Brierley JD, Cooper DS, Haugen BR, Jonklaas J, Ladenson PW, Magner J, Robbins J, Skarulis MC, Steward DL, et al. Recurrence after treatment of micropapillary thyroid can cer. Thyroid. 2009; 19:1043-1048.

19. Lin HW, Bhattacharyya N. Survival impact of treatment options for papillary microcarcinoma of the thyroid. Laryngoscope. 2009; 119:1983-1987.

20. Lang BH, Wong IO, Wong KP, Cowling BJ, Wan KY. Risk of second primary malignancy in differentiated thyroid carcinoma treated with radioactive iodine therapy. Surgery. 2012; 151:844-850.

21. Cooper DS, Doherty GM, Haugen BR, Kloos RT, Lee SL, Mandel SJ, Mazzaferri EL, McIver B, Pacini F, Schlumberger M, Sherman SI, Steward DL, Tuttle RM. Revised American Thyroid Association management guidelines for patients with thyroid nodules and differentiated thyroid cancer. Thyroid. 2009; 19:1167-1214. 
22. Barney BM, Hitchcock YJ, Sharma P, Shrieve DC, Tward JD. Overall and cause-specific survival for patients undergoing lobectomy, near-total or total thyroidectomy for differentiated thyroid cancer. Head Neck. 2011; 33:645-649.

23. Shindo M, Wu JC, Park EE, Tanzella F. The importance of central compartment elective lymph node excision in the staging and treatment of papillary thyroid cancer. Arch Otolaryngol Head Neck Surg. 2006; 132:650-654.
24. White ML, Gauger PG, Doherty GM. Central lymph node dissection in differentiated thyroid cancer. World J Surg. 2007; 31:895-904.

25. Pisello F, Geraci G, Lo Nigro C, Li Volsi F, Modica G, Sciumè C. Neck node dissection in thyroid cancer. A review. G Chir. 2010; 31:112-8. 